\title{
Preliminary experimental investigation of boundary layer in decelerating flow
}

\author{
O. Hladík ${ }^{1}$, P. Jonáśs ${ }^{1}$, and J. Př́ihoda ${ }^{1}$ \\ ${ }^{1}$ Institute of Thermomechanics AS CR, v.v.i., Praha, Czech Republic
}

\begin{abstract}
Investigations of characteristics of turbulence inside boundary layer under decelerating flow were studied by means of constant temperature anemometer. The decelerating flow was simulated in the closed circuit wind tunnel $0.9 \mathrm{~m} \times 0.5 \mathrm{~m}$ at IT AS CR. The free stream turbulence was either natural or risen up by square mesh plane grid. The details of experimental settings and measurement procedures of the instantaneous longitudinal velocity component are described and the distributions of intensity, skewness and kurtosis of turbulent fluctuations are discussed in the contribution.
\end{abstract}

\section{Introduction}

Since 1992, problem of by-pass transition is systematically studied at the Institute of Thermomechanics AS CR, v.v.i. (IT) [1]. Interesting and beneficial original notions were received on the effect of free stream turbulence (FST) intensity and length scale, surface roughness and their joint action on the moving the start of transition towards the leading edge (LE), on the shortening the transition region and on the conservation of a piece with pseudo-laminar flow just at LE. Experimental investigations were performed solely in zero pressure gradient boundary layers. The main results and conclusions were presented e.g. [2]. Regions with decelerating flow frequently occur in industrial machinery flows. Thus understanding the effect of an adverse pressure gradient on the by-pass transition seems very important. Owing to this, it was decided to extend the transition research at IT to check the validation of the till nowadays received knowledge in decelerating boundary layers.

Still unpublished measurements in turbulent boundary layer under decelerating turbulent free stream were done in the IT several decades ago [3, 4]. Critical analyse and check of the evaluation were done as to reach design fundamentals and images on specific demands on measuring methods for the upcoming continuation of bypass transition investigations. The revised results of the above mentioned experiments are briefly presented and discussed.

\section{Experimental apparatus and procedure}

The experiments were carried out in the close type wind tunnel of the IT. The investigated boundary layers were developing on a smooth flat plate located in the closed test section (height: $0.5 \mathrm{~m}$; width: $0.9 \mathrm{~m}$; length: $2.5 \mathrm{~m}$ ). The scheme of the experimental set up is shown in the figure 1 .

The plate has an elliptic leading edge and it is fastened about $0.15 \mathrm{~m}$ above lower wall of test section. The transition sandpaper-belt (grits 60; width: $0.05 \mathrm{~m}$ ) is glued on the plate $0.1 \mathrm{~m}$ downstream from the LE as to stabilize the laminar turbulent transition location. A flap (chord: $0.075 \mathrm{~m}$; deviation: $10^{\circ}$ ) is attached at the plate's trailing edge as to remove the circulation round the plate. The streamwise pressure gradient is imposed on the flat plate boundary layer by a displacement body fastened to the upper wall. The flow acceleration begins $0.15 \mathrm{~m}$ downstream LE. The flow velocity is reaching maximal value $31 \mathrm{~m} / \mathrm{s}$ in the cross section $0.45 \mathrm{~m}$ downstream LE and the flow deceleration starts $0.75 \mathrm{~m}$ downstream LE in the section $x=0$. The plane diffuser (length $1 \mathrm{~m}$; opening angle: $11.3^{\circ}$ ) has the deflected wall made from fine woven screen. Suction through this wall prevents flow separation in the diffuser. More details are given in the caption of figure 1. Similar modelling of decelerating flow is still employed e.g. Marxen et al. [5] and Uruba [6].

Pitot-static tube and Pitot tube are main devices in the mean flow characteristics measurements. Preston tube serves for the skin friction measurements. All turbulence measurements were carried out by using the linearized DISA $55 \mathrm{M}$ constant temperature anemometer system 


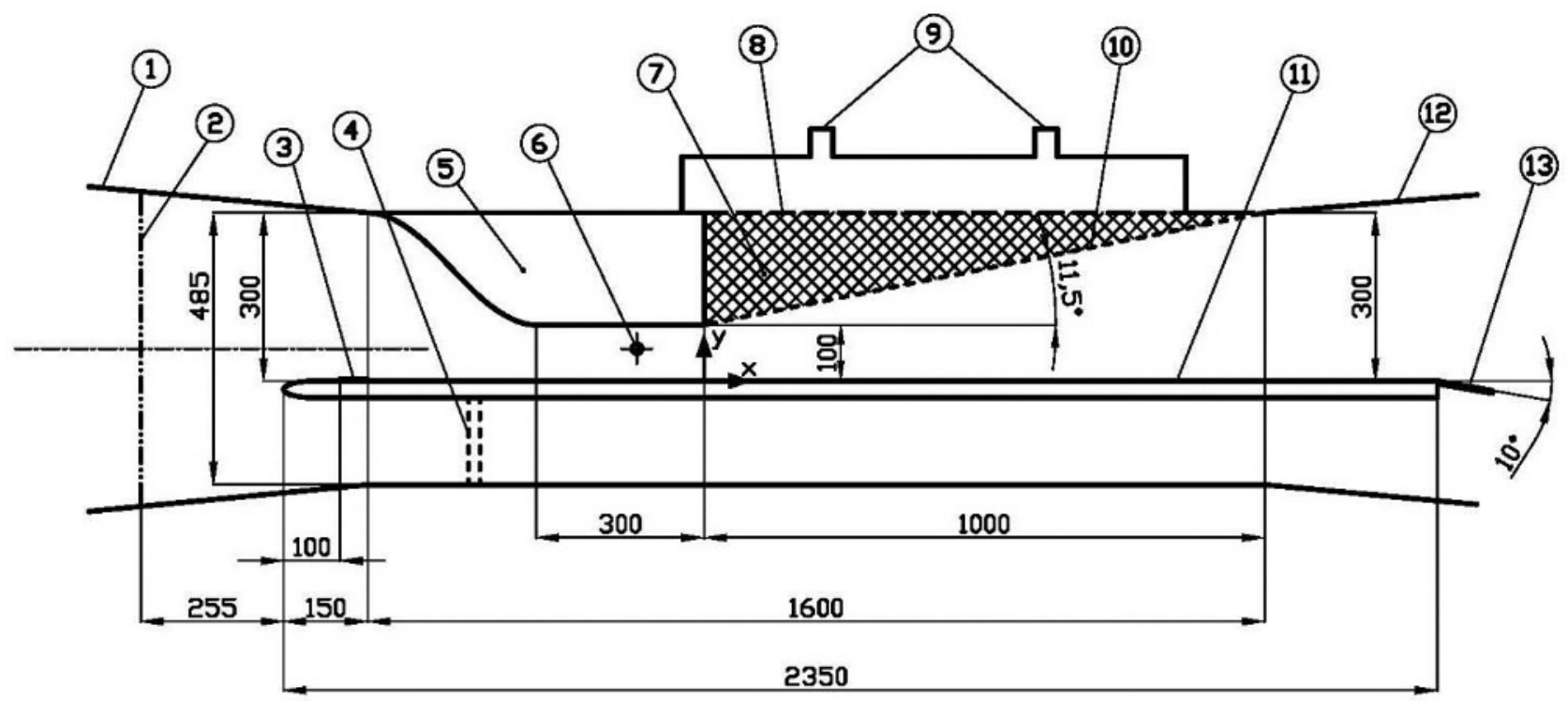

Fig. 1. Scheme of the experimental set up: 1. contraction, 2. turbulent generator, 3. sandpaper belt, 4. pressure drop generators, 5.displacement body, 6. Pitot-static tube, 7. porous material, 8. perforated wall, 9. suction, 10. woven screen plane diffuser, 11. smooth flat plate, 12. diffuser, 13. flap

with tungsten single wire probes (diameter $5.10^{-6} \mathrm{~m}$, length about $0.8 \mathrm{~mm}$ ). The CTA system was expanded by additional devices namely the amplitude analyser and correlator DIDAC 4000. Conventional turbulence characteristics were evaluated by using methods with time averaging in the framework of linearized theory. Following definitions are introduced:

intensity of the longitudinal velocity fluctuations

$$
I u[\%]=100 \sqrt{\overline{u^{2}}} / \bar{U}_{e}
$$

skewness factor $S_{u}$ and flatness factor $F_{u}$

$$
S_{u}=\overline{u^{3}} /\left(\overline{u^{2}}\right)^{3 / 2} ; \quad F_{u}=\overline{u^{4}} /\left(\overline{u^{2}}\right)^{2}
$$

skewness factor $S_{u}$, and flatness factor $F_{u}$, of the velocity fluctuations derivative are calculated using the frozen turbulence approximation and definitions (2)

$$
u^{\prime}=\partial u / \partial x=-(\partial u / \partial t) / \bar{U}
$$

\section{Turbulent free stream flow}

The free stream velocity decrease with the distance $\mathrm{x}$ from the start of the flow deceleration is shown in figure 2. The course can be described by the same empirical formulae:

$$
U_{e}(x) / U_{0}=1-0.33 x \pm 0.004, \quad[x]=m
$$

in the set up with natural turbulence as well as in the case with turbulence amplified by means of the grid generator.
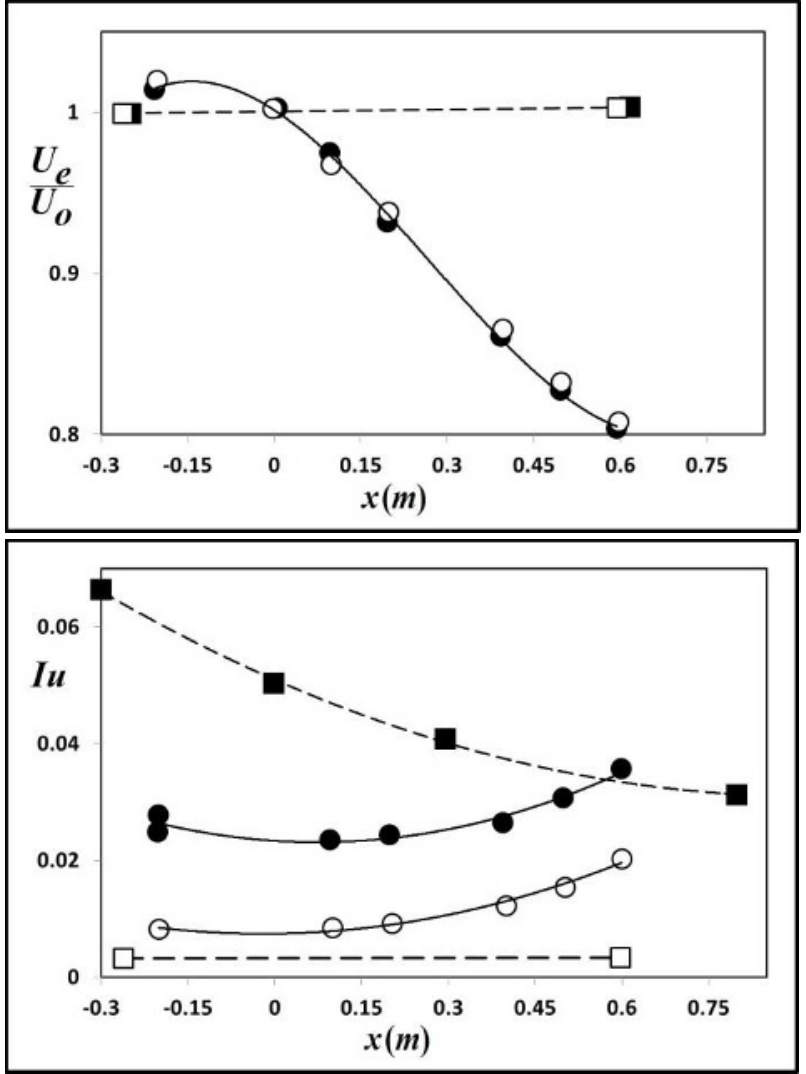

Fig. 2. Distributions of the dimensionless free stream mean velocity $U_{e} / U_{0}$ and the intensity $I u$ of the longitudinal component of turbulent velocity fluctuations in free stream; zero pressure gradient (squares), decelerated flow (circles); empty symbols - natural turbulence level, black symbols - grid turbulence. 


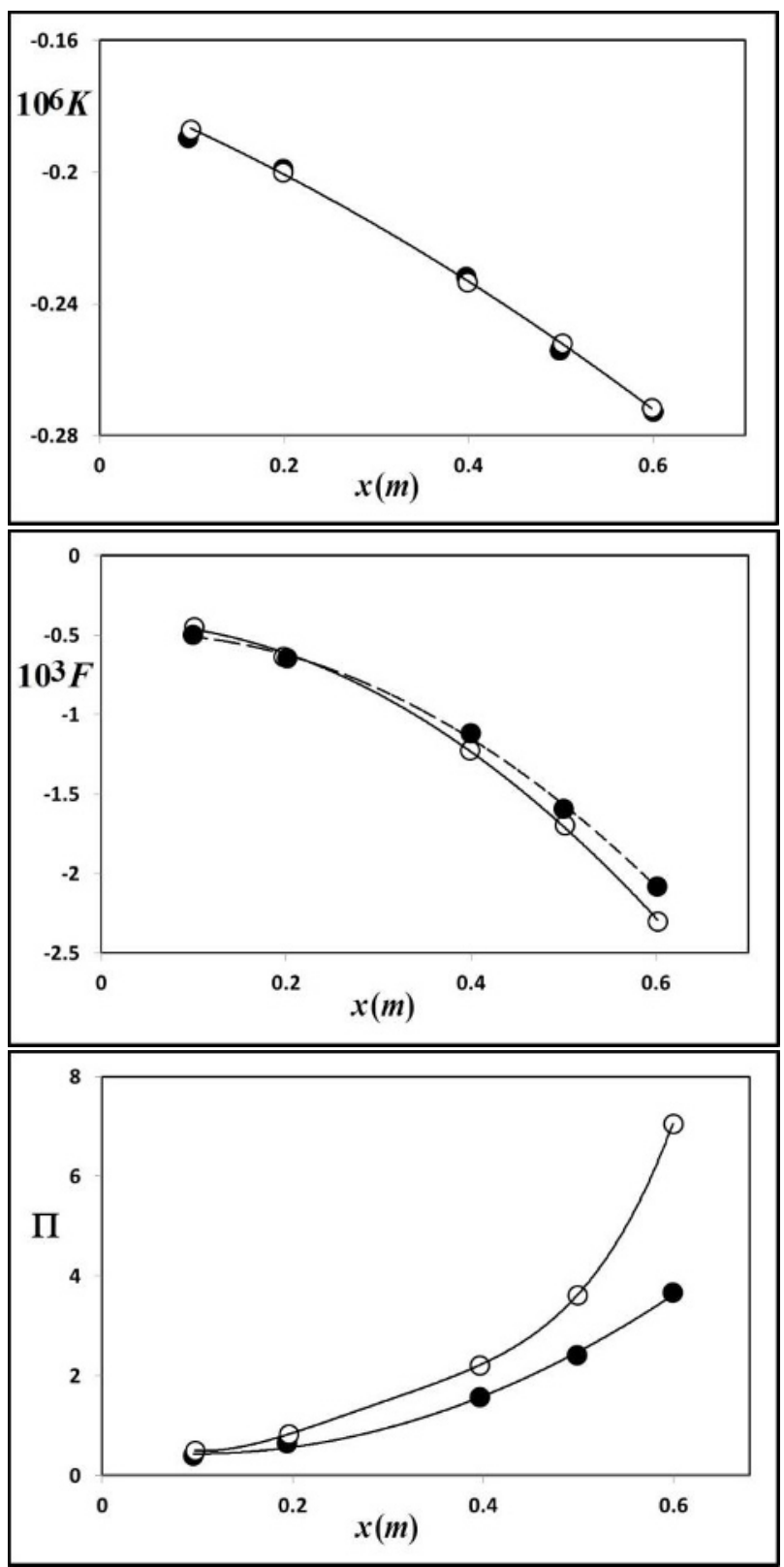

Fig. 3. Dimensionless pressure gradient parameters (symbols as in figure 2)

Distributions of three pressure gradient parameters often employed are plotted in the figure 3 . Their definitions are as follows:

$$
K=\frac{v}{U_{e}^{2}} \frac{d U_{e}}{d x} ; F=\frac{\delta_{2}}{U_{e}} \frac{d U_{e}}{d x} ; \Pi=\frac{\delta_{1}}{\tau_{w}} \frac{d P_{e}}{d x}
$$

where $v$ is kinematic viscosity, $\delta_{2}$ boundary layer momentum thickness, $\delta_{1}$ displacement thickness and $\tau_{w}$ skin friction.

Sometimes are beneficial following relations:

$$
F=K \cdot \operatorname{Re}_{2} ; \quad \Pi=\frac{2 . H_{12}}{C_{f}} F
$$

where $H_{12}=\delta_{1} / \delta_{2}$ is shape factor and $C_{f}$ skin friction coefficient.
The FST intensity $I u$, of the longitudinal component of turbulent velocity fluctuations is increasing downstream in both investigated configurations (figure 2). The main reasons for these developments are the actions of relatively thick shear layers attached at the porous suctioned wall (see figure 1, item 9). This shear layers are evident from the mean velocity profiles across the diverging 2D-channel, shown in figure 4 . Thus turbulent energy production survives at the mean flow energy expense.

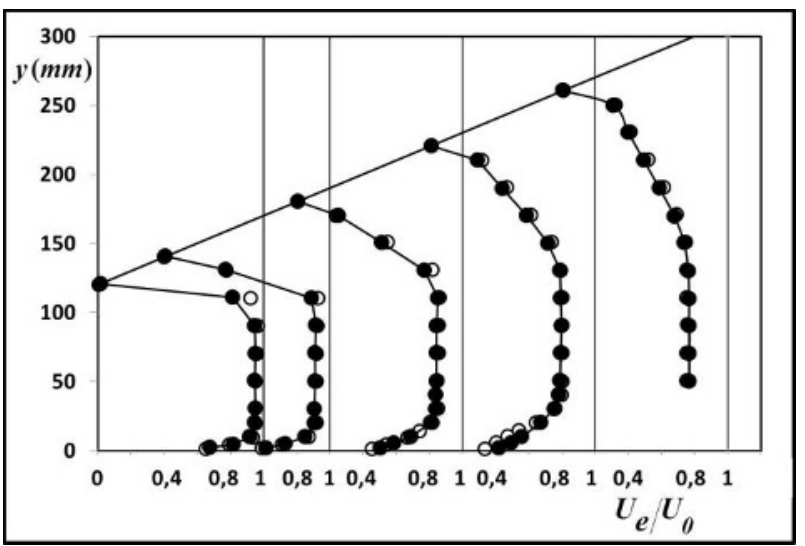

Fig. 4. Mean velocity profiles across the diverging 2D-channel. (symbols as in figure 2)

Not only turbulence intensity was evaluated from measurements but also central moments of $3^{\text {rd }}$ and $4^{\text {th }}$ order of the velocity turbulent fluctuations were calculated and similarly relevant moments of the velocity time derivative were determined. Canonical definitions are applied of the skewness factor $S_{u}$, the flatness factor $F_{u}$ and the same factors were calculated for the velocity derivative fluctuations $S_{u}$, and $F_{u}$. The flatness factor of the velocity time derivative was utilized after Rotta [7] and Townsend [8] to estimate the intermittency factor (ratio of time with occurrence turbulence to the total time of measurement):

$$
\gamma=A(\text { const. }) / F_{u^{\prime}} ; \quad F_{u^{\prime}}=\overline{e^{4}} /\left(\overline{e^{\prime 2}}\right)^{2}
$$

Resulting distributions are shown in the figure 5 . Skewness of velocity fluctuations $S_{u}$ is zero in both configurations (in the limits of measuring accuracy) up to about $x=0.4 \mathrm{~m}$. Then it decreases up to -0.4 in natural FST and 0.2 in grid FST. Having in mind also the weakly positive value of kurtosis $F_{u}$ (about 3.2) the turbulent diffusion across the main stream is obvious.

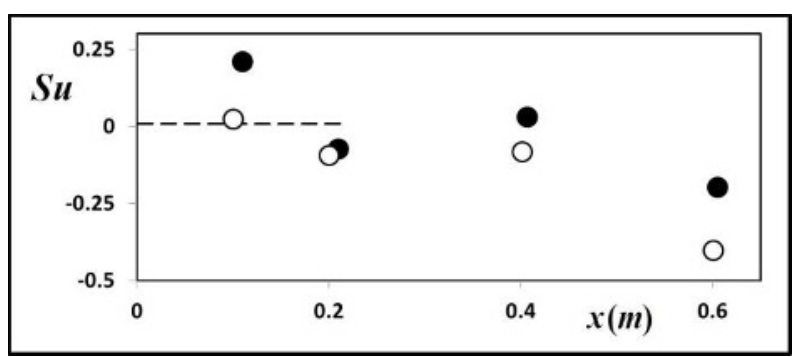




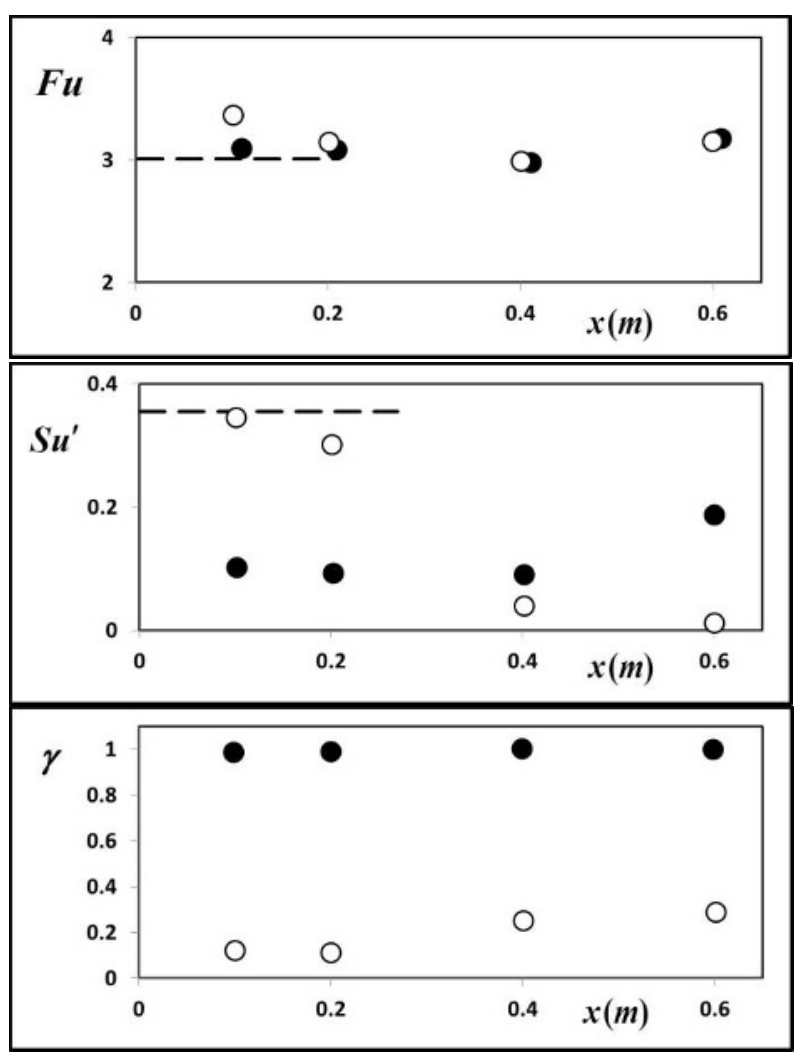

Fig. 5. Normalized central moments of $3^{\text {rd }}$ and $4^{\text {th }}$ order of longitudinal velocity $S_{u}$ and $F_{u}$ and the skewness of velocity derivative $S_{\mathrm{u}^{\prime}}$ and the estimate of intermittency factor $\gamma$ (dashed lines represent values in flow behind the grid).

In natural FST conditions, the skewness factor of the velocity gradient fluctuations, $d u / d x$, (Taylor approximation of frozen flow is applied in calculation) is stream wise decreasing from 0.38 at $x<0.3 \mathrm{~m}$ up to zero in natural FST. Equal values as at $x<0.3 m$ quoted Townsend [8] from measurements in the wake and downstream a grid. Possibly a small flow separation occurred upstream the diffuser entry. The distribution of the intermittency factor signifies the streamwise growing intermittency of natural FST up to $\gamma=0.3$.

In grid turbulence the skewness and flatness distributions of turbulent velocity fluctuations are roughly similar to those being already discussed. From the distributions of skewness and flatness shown in figure 5 follows that the probability density function of the velocity derivative fluctuations is little asymmetric with the higher rate of positive fluctuations. Perhaps the convective transport dominates above the lateral one. Further the grid FST is fully turbulent downstream the turbulence generating grid and turbulence energy is stream wise increasing from the diffuser entry $x=0$.

\section{Main features of investigated turbulent boundary layers}

Distributions of boundary layer thickness $\delta$, the shape factor $H_{12}=\delta_{1} / \delta_{2}$ and the skin friction coefficient $C_{f}$ are plotted in the figure 6 as functions of the distance $\mathrm{x}$ from the diffuser entry, section $x=0$. Presented results [3] confirm the knowledge of the free stream turbulence effect on turbulent boundary layer namely: the more rapid thickening of boundary layer under amplified FST (upper part of figure 6); the more cambered mean velocity profiles i.e. lower values of the shape factor $H_{12}$ (middle part of figure 6) sign of the stabilizing effect of free stream turbulence and the increase of wall friction with increasing turbulence level at the given Reynolds number (bottom graph in figure 6). All mentioned effects are resulting from the amplification of turbulent diffusion across the layer.

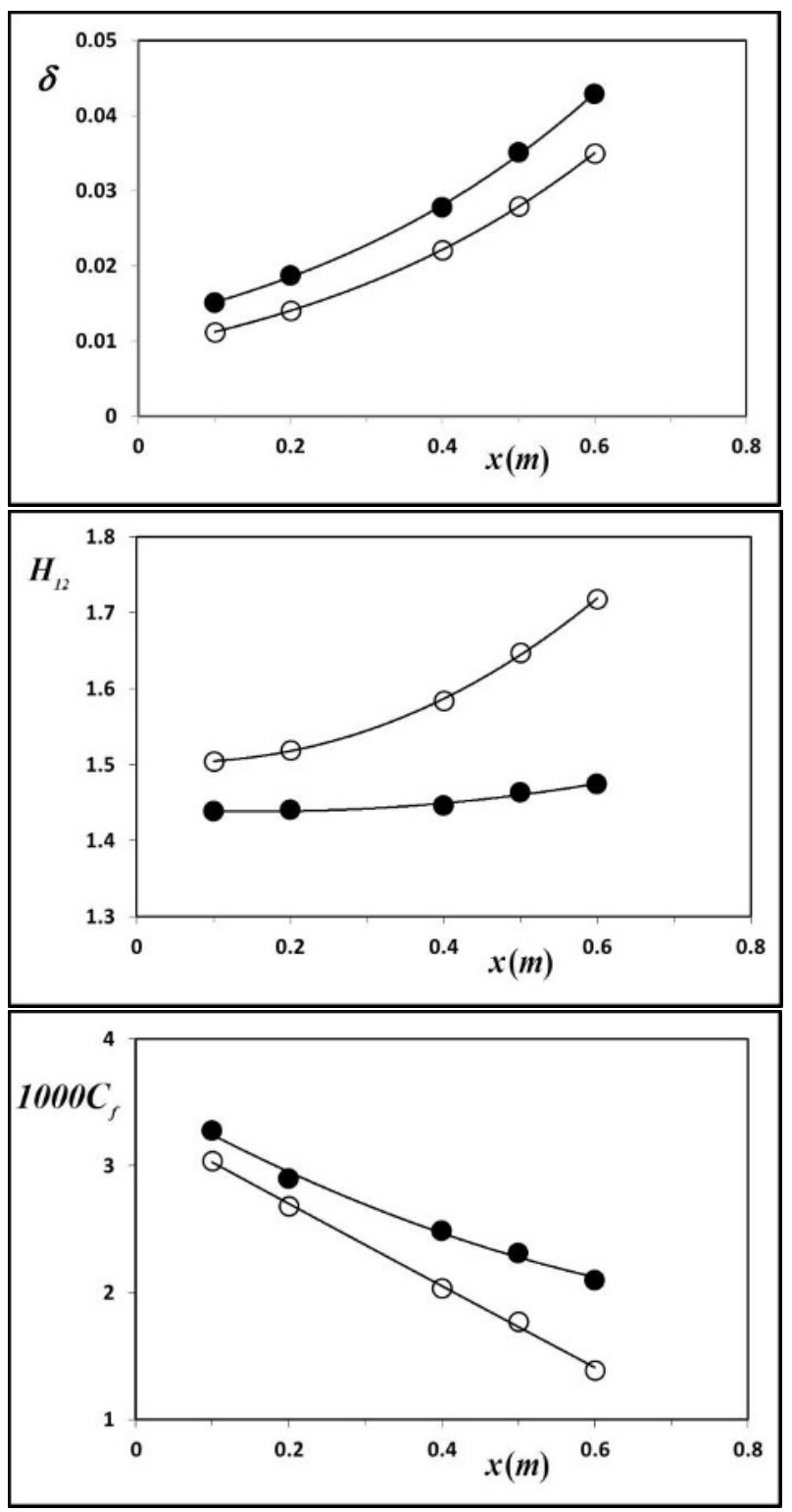

Fig. 6. Important characteristics of turbulent boundary layers developing in natural FST (empty symbols) and in grid FST (black symbols).

\section{Turbulence in boundary layer inner region}

The flow structures of boundary layers are characterized by characteristics plotted in figure 7 using the notation: under natural FST (empty marks) or in the grid generated FST (dark marks). Measurements were accomplished in 
four sections $x=0.1 \mathrm{~m}$ (circles); $0.2 \mathrm{~m}$ (triangles); $0.4 \mathrm{~m}$ (squares) and $0.6 \mathrm{~m}$ (rhombus). Table 1 includes additional information about figure 7 particularly momentum thickness Reynolds number and turbulence intensity beyond the turbulent boundary layer. Profiles of the standard deviation of velocity fluctuations $\sqrt{\overline{u^{2}}}$ shown in figure 7 , are normalized by the friction velocity $u_{\tau}$. The dimensionless distance from the flat plate $y^{+}$is the true $y$ normalized by the viscous length scale (fraction of kinematic viscosity and friction velocity). The courses in natural FST and grid FST are very similar, roughly the same within the accuracy limits, for $y^{+}>30$ i.e. outside the buffer layer. Amplified FST is affecting the inner part of boundary layer in decelerated flow like in the zero pressure gradient flow. The viscous sublayer becomes thinner.

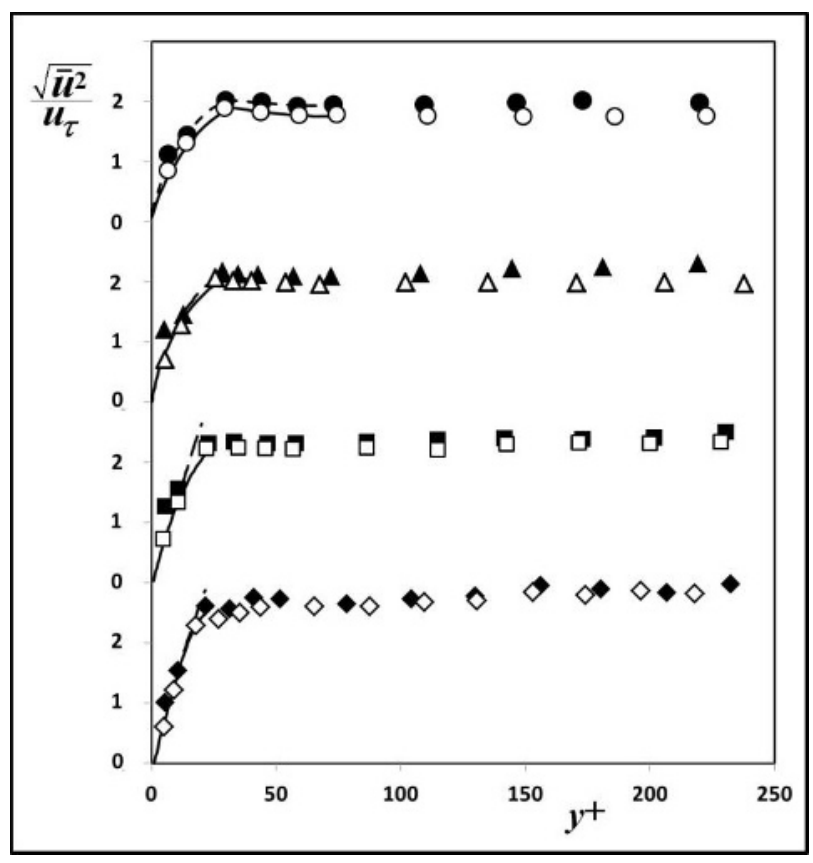

Fig. 7. Profiles of dimensionless standard deviation of velocity fluctuations normalized by inner variables (additional information in Table 1).

Table 1. Additional information to figure 7 and figure 9.

\begin{tabular}{|c|c|c|c|c|}
\hline symbol & $\mathbf{x}(\mathrm{m})$ & $10^{-3} \boldsymbol{R e}_{2}$ & $\left(\frac{\sqrt{u^{2}}}{u_{\tau}}\right)_{\infty}$ & $\left(\frac{\sqrt{u^{2}}}{U_{\infty}}\right)_{\infty}$ \\
\hline$\bigcirc$ & 0.1 & 2.47 & 0.20 & 0.85 \\
\hline $\boldsymbol{O}$ & 0.1 & 2.53 & 0.51 & 2.83 \\
\hline$\Delta$ & 0.2 & 3.22 & 0.21 & 0.92 \\
\hline $\boldsymbol{\Delta}$ & 0.2 & 3.28 & 0.61 & 2.60 \\
\hline$\square$ & 0.4 & 5.23 & 0.33 & 1.18 \\
\hline$\square$ & 0.4 & 5.05 & 0.64 & 2.49 \\
\hline $\boldsymbol{\nabla}$ & 0.6 & 8.39 & 0.68 & 2.00 \\
\hline $\boldsymbol{\nabla}$ & 0.6 & 7.56 & 1.00 & 3.52 \\
\hline
\end{tabular}

After Wallace et al. [9] the production of kinetic energy of turbulent motions reaches maximum near the inner limit of buffer region about in location $\left(y^{+} \sim 10\right)$ where skewness $S_{u}$ is changing its sign. With the growing FST the location of maximal energy production of turbulent motions moves towards the wall. Profiles of the skewness factor $S_{u}$ plotted on figure 8 confirm this statement because $S_{u}$-profiles determined in the grid FST are crossing the zero level nearer the wall, at $\left(y^{+}\right)_{g} \sim 5 \div 7$ than $S_{u}$-profiles measured in the natural FST, at $\left(y^{+}\right)_{n} \sim 10$ $\div 12$. Note: the friction velocity in the grid FST is larger than in the natural FST thus real difference of true locations $y_{n}-y_{g}$ is larger than the difference of dimensionless locations. More elaborated discussion of the kurtosis profiles and statistical characteristics the fluctuating velocity derivative are outside the scope of this contribution. More detailed discussion is given in e.g. [4].

The flow deceleration as well as the changes of turbulence level in external flow does not affect qualitatively processes in the inner layer which is the main conclusion of this chapter. The quantitative differences/effects manifest themselves in the vicinity of viscous sublayer and buffer layer interface. They are the consequence of turbulent diffusion across the flow when disturbances originating in locations with higher mean velocity are penetrating towards the wall.

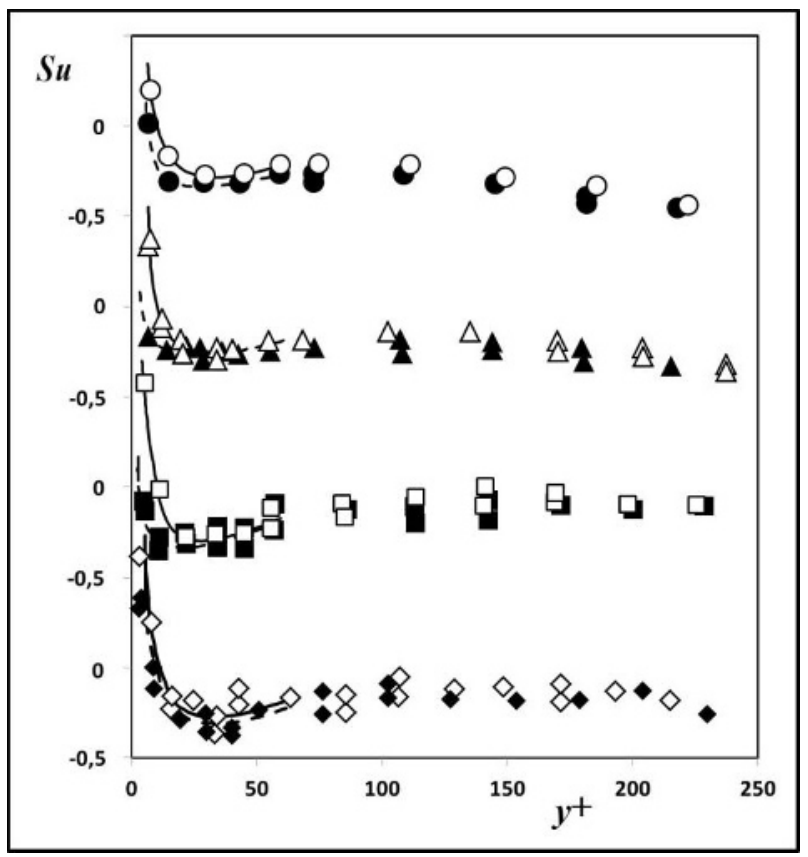

Fig. 8. Skewness factor profiles in the inner region.

\section{Turbulence in boundary layer outer region}

The advanced discussions of measurements in the outer regions of investigated layers work on the evaluated profiles of the intensity of turbulence (figure 9), the skewness factor and the flatness one of the longitudinal velocity fluctuations and on the relevant statistical characteristics of the derivative of velocity fluctuations. The profiles of the intermittency factor (figure 10 and figure 11) were evaluated after the formulae (7). 


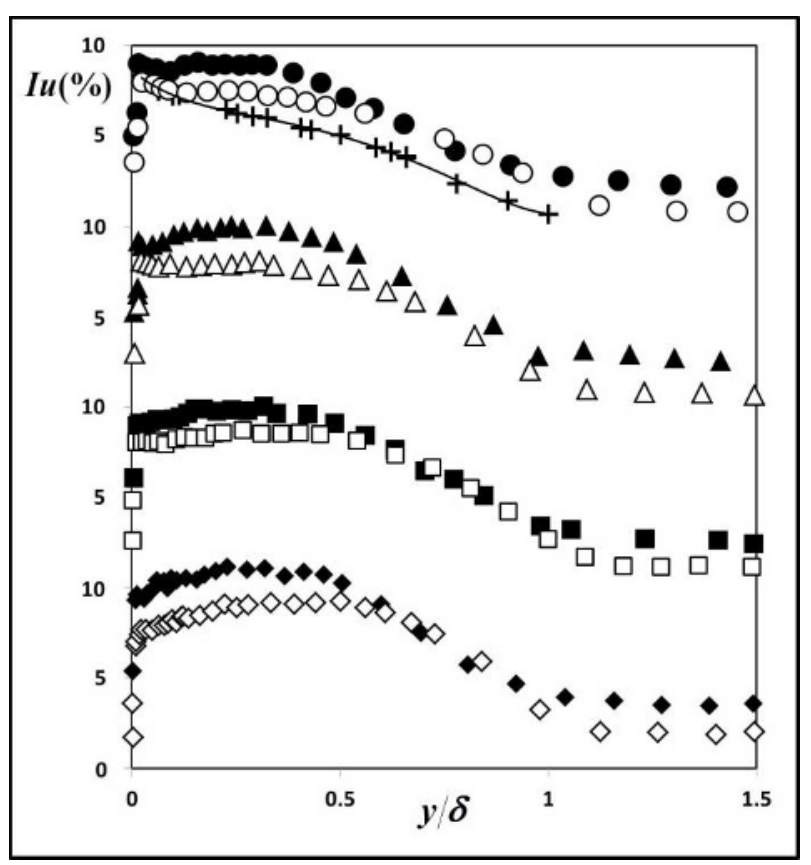

Fig. 9. Profiles of turbulence intensity $I_{u}$ i.e. profiles dimensionless standard deviation of velocity fluctuations normalized by outer variables (full line represents Klebanoff measurements). Symbols are as in figure 7 and additional information is in Table 1 .

The shape of the measured longitudinal velocity fluctuations intensity profiles $I_{u}$ (figure 9) is dissimilar to the shape of the intensity determined in the flat-plate boundary layer on smooth surface in non-turbulent free stream (data borrowed from the Klebanoff measurements presented by Hinze [10]). The flat second maximum of $I_{u}$ $(x, y)$ arises further from the surface additionally to the pointed first maximum near the surface $\left(y \sim 0.03 \delta\right.$ or $y^{+} \sim$ $15)$ if the flow become decelerating or if the free stream turbulence amplifies. The joint action of the decelerating pressure gradient and the FST amplification make the first maximum weaker. Finally the first maximum disappears. Having in mind that main portion to the turbulence kinetic energy originates from fluctuations of the velocity longitudinal component then it is possible to assume that maximum of turbulence energy moves from the surface due to the investigated effects. Next it might be surmised that maximum of the Reynolds shear stress is located in the proximity of the energy maximum because the turbulence production mechanism - conversion of the mean flow kinetic energy to turbulence. Simultaneously the Reynolds stresses transfer momentum across the boundary layer to the surface and thus the equilibrium of forces affecting fluid particles. Additionally to viscous forces the pressure gradient inhibits fluid particles in the decelerating external flow. Therefore momentum transfer to the surface reduces retardation of fluid particles, decreases the skin friction coefficient $C_{f}$ (figure 6) and postpones the flow separation.

Profiles of skewness $S_{u}$ and flatness $F_{u}$ indicate that the FST turbulence level only little affect the flow in the region from the surface $y=0$ up to about the middle of boundary $y \sim \delta / 2$. Further from these profiles follows that turbulence structures of boundary layers in natural FST and grid FST are different in the vicinity of boundary limit. This results from the fact that turbulent motions born inside the layer must bind to the structure of the free stream. In particular case - non-turbulent free stream the vorticity generated in the layer must fully decay inside the Corrsin superlayer.

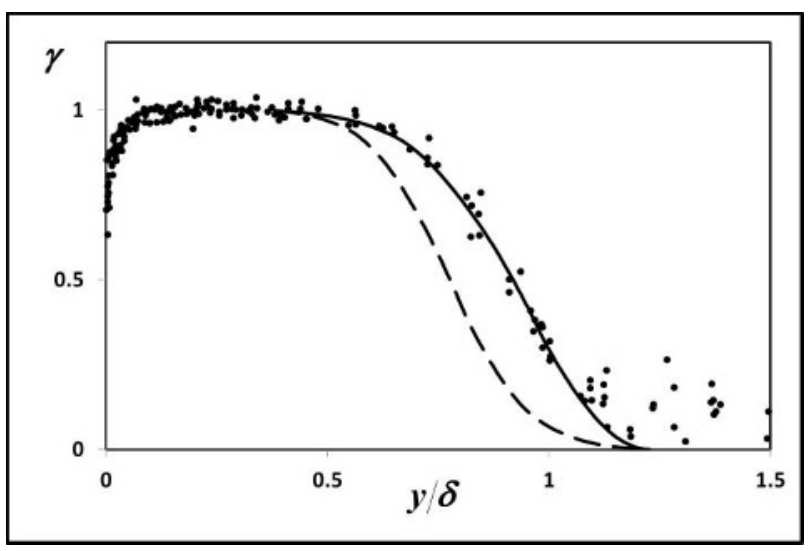

Fig. 10. Profile of the intermittency factor across turbulent boundary layer in natural FST (dashed line represents course determined by Klebanoff).

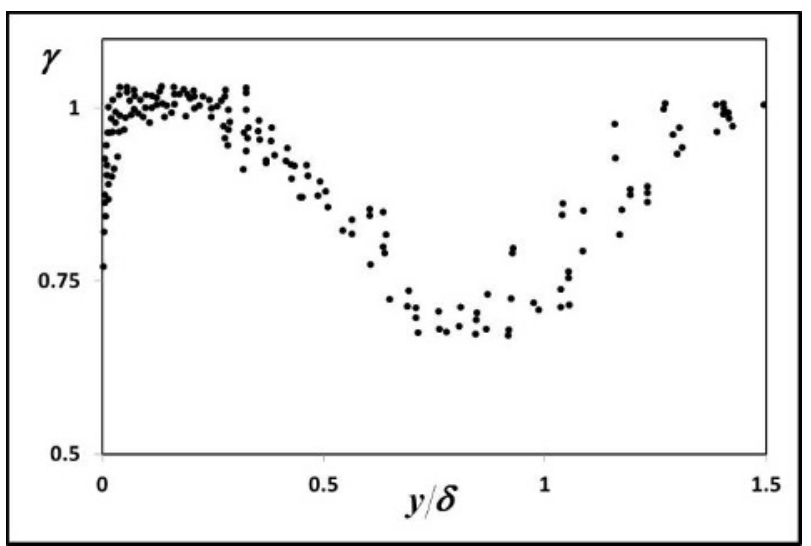

Fig. 11. Profile of the intermittency factor across turbulent boundary layer in grid generated FST.

Both investigated boundary layers are under turbulent FST but the natural turbulence is obviously of an intermittent character and the grid FST structure is near the isotropic turbulence structure. The different action of the studied events is obvious from figure 10 and figure 11 , where the profiles of the intermittency factor (7) are shown. The points measured in different sections $x=$ const. are not distinguished in these figures for their better transparency. Owing to intermittent character of natural FST and intensified momentum transfer across the boundary layer the intermittency factor profile in figure 10 slowly and incompletely approaches the zero value in comparison with the course determined by Klebanoff in the smooth flat plate boundary layer under non-turbulent flow (reproduced in e.g. [10]).

Wholly different shape than in the figure 10 has the intermittency profile across turbulent boundary layer measured in the grid generated FST plotted in figure 11. The decrease of evaluated intermittency, from $\gamma=1$ up to $\sim 0.7$ in the zone between $y / \delta=0.2$ up to 1.4 is 
surprising. It is surprising namely that the parameter in the numerator of the formulae (7) had constant value for the whole family of profiles in figure 11. Possibly this indicates a mixing process among the turbulent structures generated in the inner layer and in the structures coming from the free stream.

\section{Conclusions}

The presented results agree with the knowledge received from investigations of the effect of the free stream turbulence (FST) on the structure of a flat-plate turbulent boundary layer i.e. they are valid also in turbulent decelerating flow

- the distribution of turbulent boundary layer in the inner layer and the outer layer remains;

- the mechanism of turbulent processes in the inner layer is not influenced by FST;

- the effect of FST proves namely in the outer layer where turbulence born deep inside the inner layer is binding with the FST;

- the alterations due to increasing FST make more stable shape of the velocity profiles, cause an increase in wall friction and postpone a flow separation.

The experimental layout with the displacement body at the roof of the working section proves successful but it was necessary to prevent boundary layer separation on the deflected wall. The layer was sucked off.

\section{Acknowledgement}

This work has been supported by the Czech Science Foundation, the project GAP101/12/127 and No.103/09/0977.

\section{References}

1. P. Jonáš, ZAMM - Z. angew. Math. Mech. 75 S1, 339-340 (1995)

2. Hladík, P. Jonáš, O. Mazur and V. Uruba, ETC 13. Journal of Physics, Conf. Series 318, 032030, 1-19 (2011)

3. J. Př́hoda, (In Czech). Rep. Institute of Thermomechanics CAS, Prague, Z-744/81 (1980)

4. P. Jonáš, (In Czech). Rep. Institute of Thermomechanics CAS, Prague, Z-761/81 (1980)

5. O. Marxen, M. Lang, U. Rist and S. Wagner, Flow, Turbulence and Combustion 71, 133-146 (2003)

6. V.Uruba, M.Knob. Proceedings in Applied Mathematics and Mechanics, Roč. 8, č. 1, s. 10021-10024 (2008)

7. J.C. Rotta, Progress in Aeronautical Sci. 2, Pergamon Press, Macmillan Co. New York (1962)

8. A.A. Townsend, Cambridge University Press (1956)
9. J.M. Wallace and R.S. Brodkey, Max-PlanckInstitut f. Strömungsforschung, Bericht 108/1976, Göttingen (1976)

10. J.O. Hinze, Turbulence. McGraw Hill, New York (1975) 